\title{
Use of Counter-balanced Smith Machine Affects Performance Measurements for Rebound Bench Press Throws
}

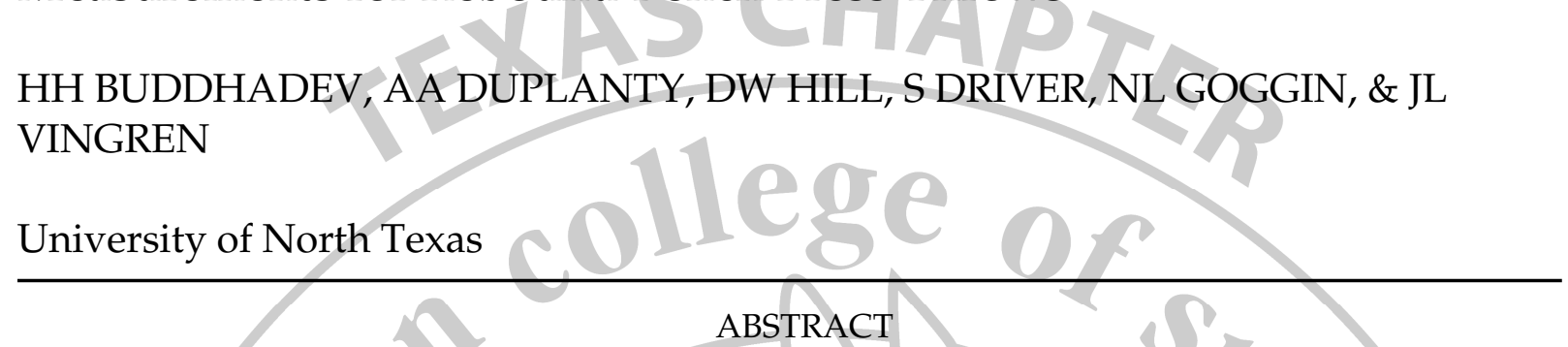

Rebound bench presses throws (RBT), often performed on a Smith machine, are used for assessment and training of upper body power. During a RBT, the stretch-shortening cycle potentiates performance in the concentric movement. Smith machines frequently utilize a counter-balance weight to reduce the net load on the barbell; however, the use of counter-balance weight affects measures of performance for RBT. PURPOSE: To evaluate how the use of a counter-balanced Smith machine affects performance measures for RBT. METHODS: Performance measures for the no counter-balanced (NCB) and counter-balanced (CB) RBT were assessed for 24 men (age: $23 \pm 3$ years, height: $179 \pm 6 \mathrm{~cm}$, mass: $91 \pm 17 \mathrm{~kg}$, bench press 1repetition maximum [1RM]: $107 \pm 18 \mathrm{~kg}$ ). Each participant performed 2 sets of 2 repetitions of RBT for each condition at $30 \%$ of their 1RM. Peak power, peak force, peak concentric and eccentric velocities, and duration of eccentric and concentric phases were measured using a linear accelerometer attached to the barbell; peak ground reaction force (GRF) was measured using a force plate. For each condition, data from the repetition with the highest peak power was used in further analyses. Peak EMG was measured for the right pectoral, deltoid and triceps muscles and normalized using peak EMG in the 1RM. RESULTS: Peak barbell measurements for power (NCB: $1220 \pm 269 \mathrm{~W}, \mathrm{CB}: 1069 \pm 255 \mathrm{~W}$ ), force (NCB: $906 \pm 252 \mathrm{~N}, \mathrm{CB}: 713 \pm 143 \mathrm{~N}$ ), and concentric (NCB: $2.54 \pm 0.27 \mathrm{~m} \cdot \mathrm{s}^{-1}, \mathrm{CB}: 2.24 \pm 0.32 \mathrm{~m} \cdot \mathrm{s}^{-1}$ ) and eccentric (NCB: $\left.-1.19 \pm 0.46 \mathrm{~m} \cdot \mathrm{s}^{-1}, \mathrm{CB}:-0.95 \pm 0.29 \mathrm{~m} \cdot \mathrm{s}^{-1}\right)$ velocities were significantly $(p<0.05)$ higher for NCB compared to $\mathrm{CB}$. The durations for the eccentric (NCB: $0.53 \pm 0.16 \mathrm{~s}, \mathrm{CB}: 0.64 \pm 0.12 \mathrm{~s}$ ) and concentric phases (NCB: $0.58 \pm 0.58 \mathrm{~s}, \mathrm{CB}: 0.77 \pm 0.82 \mathrm{~s}$ ), and peak pectoral EMG (NCB: $91 \pm 21 \%$ of 1RM, CB: $101 \pm$ $24 \%$ of $1 R M$ ) were lower for NCB compared to CB. Peak EMG for deltoid and triceps and peak GRF were unaffected by the use of counter-balance weights. CONCLUSION: The use of CB equipment resulted in reduced performance measurements (peak power, peak force, and peak eccentric and concentric velocities) for the RBT compared to NCB equipment. The lower peak eccentric stretch velocity likely resulted in a less effective stretch-shortening cycle for $\mathrm{CB}$ compared to $\mathrm{NCB}$ and thus helps explain the lower performance measurements found for CB.

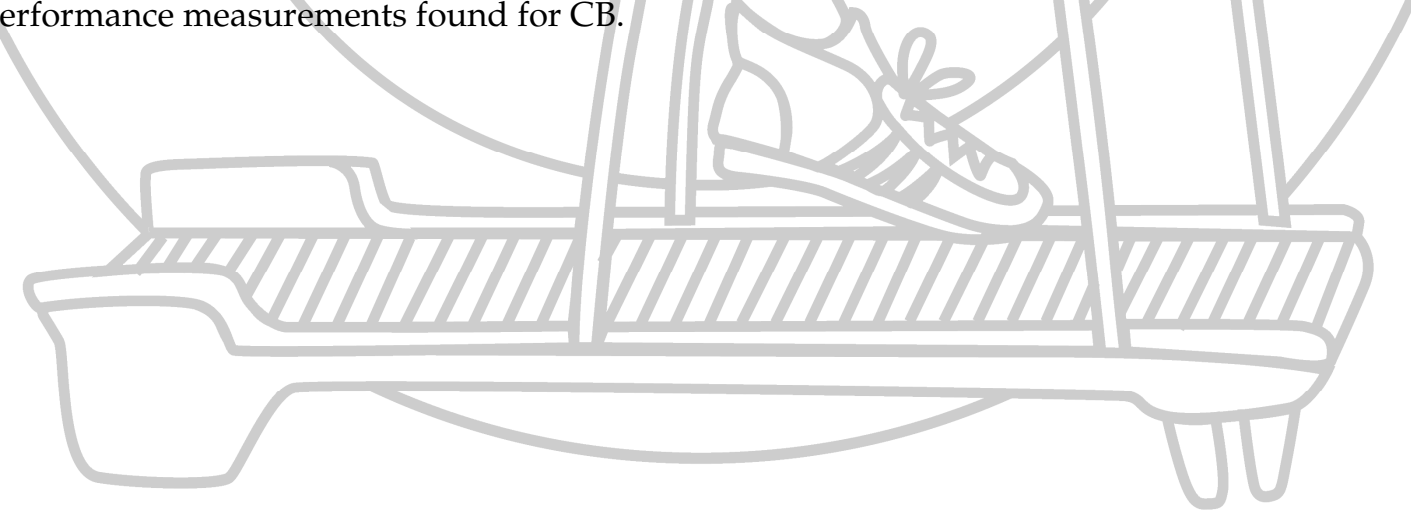

